\title{
Dynamic monitoring of EGFR mutations in circulating cell-free DNA for EGFR-mutant metastatic patients with lung cancer: Early detection of drug resistance and prognostic significance
}

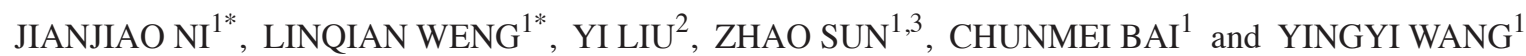 \\ ${ }^{1}$ Department of Medical Oncology, Peking Union Medical College Hospital, Peking Union Medical College and \\ Chinese Academy of Medical Sciences, Beijing 100730; ${ }^{2}$ Translational Medicine Center, Laboratory of Oncology, \\ Affiliated Hospital of Academy of Military Medical Sciences, Beijing 100071; ${ }^{3}$ Institute of Basic Medical Sciences, \\ Peking Union Medical College and Chinese Academy of Medical Sciences, Beijing 100730, P.R. China
}

Received March 31, 2016; Accepted February 13, 2017

DOI: $10.3892 / \mathrm{ol} .2017 .6022$

\begin{abstract}
Detecting genetic mutations in circulating cell-free DNA (cfDNA) is a promising approach of liquid biopsy. Between June 2014 and May 2015, 168 plasma samples were collected monthly from 20 patients with metastatic lung adenocarcinoma with epidermal growth factor receptor (EGFR) mutation receiving gefitinib therapy. Clinically relevant EGFR mutations, including exon 19 deletion, L858R and T790M, were quantified using droplet digital polymerase chain reaction. In baseline samples, 19 (95.0\%) patients had the same mutation with the matched tumors, and pretreatment T790M mutations were also detected in $3(15.0 \%)$ patients. The dynamics of EGFR mutations were generally associated with treatment response for patients with or without measurable disease. For patients with immeasurable tumor deposits, monitoring disease evolution using cfDNA-based mutation quantification appeared to be more reliable compared with measuring the diameters of target tumor lesions. In addition, molecular progressive disease, defined as a $\geq 20 \%$ increase of EGFR mutation concentration compared with the lowest concentration recorded during treatment, was tracked up to 8 months prior to objective progression. In survival analysis, sex $(\mathrm{P}=0.005)$, pretreatment $\mathrm{T} 790 \mathrm{M}$ mutation status $(\mathrm{P}=0.006), \mathrm{T} 790 \mathrm{M}$ mutation status at the disease progression $(\mathrm{P}=0.043)$ and growth rate of EGFR mutations $(\mathrm{P}=0.023)$, had a significant impact on median progression-free survival. In conclusion,
\end{abstract}

Correspondence to: Professor Yingyi Wang, Department of Medical Oncology, Peking Union Medical College Hospital, Peking Union Medical College and Chinese Academy of Medical Sciences, 1 Shuai-Fu-Yuan, Beijing 100730, P.R. China

E-mail: pumch_oncology@hotmail.com

${ }^{*}$ Contributed equally

Key words: lung cancer, epidermal growth factor receptor, circulating cell-free DNA, digital polymerase chain reaction dynamic monitoring of EGFR mutations in cfDNA is feasible and appears to be useful in early prediction of drug resistance for patients with lung cancer receiving EGFR tyrosine kinase inhibitors.

\section{Introduction}

Gefitinib is widely used for patients with lung adenocarcinoma in Asia harboring sensitizing epidermal growth factor receptor (EGFR) mutations, and usually results in a high response rate and prolonged progression-free survival (PFS) (1-3). However, almost every patient ultimately develops drug resistance following a median duration of $\sim 12$ months (1-3). A secondary T790M point mutation is the underlying mechanism in $\sim 60 \%$ of patients whose disease progresses beyond the first generation of EGFR tyrosine kinase inhibitors (TKIs) (4-6). Novel TKIs have been developed that specifically target the T790M mutation, including AZD9291 (7) and CO-1686 (8). Therefore, creating a reliable and cost-effective approach for the real-time monitoring of EGFR mutations is of great importance.

Collection of serial re-biopsy tissues in routine clinical practice is not always feasible, and thus, circulating cell-free DNA (cfDNA) extracted from patient plasma has been proposed as a promising alternative (9-11). Numerous cutting-edge platforms have been developed $(12,13)$, and droplet digital polymerase chain reaction (ddPCR) is one of the most accurate and robust methods for absolute nucleic acid quantification (13). Previous studies using these platforms have demonstrated a sensitivity and specificity $>90 \%$ for detecting EGFR mutations in cfDNA (14-17). Notably, early detection of T790M mutation up to 16 weeks prior to radiographic progression was shown in a proof-of-concept study that collected a fraction of representative plasma samples in each patient (15). However, this result requires confirmation in larger studies with consecutive samples from a more homogeneous patient population with a predefined and unified time interval. In addition, the growth rate of tumor burden represented by tumor size or tumor volume was found to be associated with patient survival (18). However, the prognostic 
significance of parameters derived from cfDNA-based EGFR mutation quantification, including the growth rate of EGFR mutations, has not been investigated.

In order to explore the association between the dynamics of EGFR mutations in cfDNA and disease evolution, 20 patients with EGFR-mutant advanced lung adenocarcinoma receiving first-line gefitinib therapy were prospectively enrolled in the present study. Clinically relevant activating and resistant EGFR mutations in monthly collected samples were quantified using ddPCR for a duration of up to 12 months. In addition, the prognostic significance of pretreatment T790M mutation status, as well as the growth rate of EGFR mutations in cfDNA, were examined.

\section{Materials and methods}

Patient population. Patients with metastatic lung adenocarcinoma receiving first-line gefitinib therapy and meeting the following criteria were prospectively enrolled. Firstly, patients must harbor one of the two most common sensitizing EGFR mutations, exon 19 E746-A750 deletion (19del) or L858R mutation (L858R), and not the T790M mutation, in their pretreatment tumor tissues. Detection of EGFR mutations in tumor tissues was performed using the Amplification Refractory Mutation System (ARMS), which was approved by the Chinese Food and Drug Administration for in vitro diagnostic use, covering the 29 most common EGFR mutations in lung cancer (17). Patients with 19del were categorized as $19 \mathrm{del}$ patients, while patients with the L858R mutation were categorized as L858R patients. Secondly, patients must have adequate baseline plasma samples and baseline tumor information, including primary and metastatic locations, size, histology and radiological images. Finally, patients must have a minimum duration of gefitinib therapy of 3 months without radiological progression (to rule out patients with primary resistance as much as possible), and consent to monthly follow-ups in the clinics of the Department of Medical Oncology of Peking Union Medical College Hospital (PUMCH; Beijing, China).

Patients with measurable or immeasurable disease were included. The present study was approved by the Institutional Review Boards of PUMCH. All patients provided written informed consent.

Sample processing and quantification of EGFR mutations. For each eligible patient, baseline blood samples were retrospectively gathered from the biobank of PUMCH and subsequent samples were collected during monthly follow-ups. The present study also collected five blood samples from five healthy donors (three males and two females, with a median age of 33-years). Each blood sample was spun into plasma by centrifugation for $10 \mathrm{~min}$ at $1,200 \mathrm{x} \mathrm{g}$ and the plasma supernatant was further cleared by centrifugation for $10 \mathrm{~min}$ at $3,000 \times \mathrm{g}$, all at $\leq 4^{\circ} \mathrm{C}$ for $2 \mathrm{~h}$, and cfDNA was extracted using the QIAamp DNA blood mini kit (Qiagen $\mathrm{GmbH}$, Hilden, Germany). L858R and T790M mutations were evaluated in samples from L858R patients, while 19del and T790M mutations were evaluated in samples from 19del patients. All three mutations were evaluated in the samples from the 5 healthy donors. EGFR mutations were detected using the
PrimePCR ${ }^{\mathrm{TM}} \mathrm{ddPCR}^{\mathrm{TM}}$ Mutation Detection Assay kits (Bio-Rad Laboratories, Inc. Hercules, CA, USA; catalog nos., 1863103, 1863104 and 1863105) and the QX100 ${ }^{\mathrm{TM}}$ AutoDG $^{\mathrm{TM}}$ Droplet Digital $^{\mathrm{TM}}$ PCR system (Bio-Rad Laboratories, Inc.), according to the manufacturer's protocol. All assays were performed in triplicate and the results were reported as copies of mutant allele per $\mathrm{ml}$ of plasma, as described in a previous study (15).

Clinical data collection. Standard clinicopathological data were collected from each patient, including demographics, primary and metastatic tumor characteristics, tumor tissue genotyping results, bimonthly radiological images, treatment and response, most recent follow-up date and progression status. PFS was defined as the time interval between the beginning of gefitinib therapy and disease progression or mortality. Treatment response and disease progression were defined according to Response Evaluation Criteria in Solid Tumors 1.1 (RECIST 1.1). Taking the lowest EGFR mutation concentration recorded since the treatment started as reference, molecular progressive disease was defined as $\geq 20 \%$ increase of sensitizing EGFR mutations and/or T790M mutations, which is similar to RECIST.

Growth rate of tumor burden was calculated in each patient with measurable disease. In line with a previous study (18), it was defined as the increase of the target tumor lesion diameter during the last two follow-ups, prior to documentation of disease progression. For the 2 patients that did not experience disease progression, the last two follow-ups prior to the end of the present study were used. Growth rate of EGFR mutation concentration was calculated for each patient in the same manner and the geometric mean was calculated when growth rates of sensitizing mutation and T790M mutation could be calculated.

Statistical analysis. All analyses were performed using SPSS version 12.0 (SPSS, Inc., Chicago, IL, USA). All the data are presented as the mean \pm standard deviation. Comparisons of proportions were performed by $\chi^{2}$ tests. Survival curves for PFS were created by the Kaplan-Meier method. Log-rank tests were used to compare the survival curves between different subgroups. Pearson's test was used to determine the significance of linear correlations between different parameters. Two-tailed tests were used and $\mathrm{P}<0.05$ was considered to indicate a statistically significant difference.

\section{Results}

Characteristics of patients. A total of 20 patients were enrolled in the present study (Table I), including 16 (80\%) female and $4(20 \%)$ male individuals. The average age of the patients was $58.9 \pm 10.2$ years, with no significant difference being observed between male and female patients ( 56.2 vs. 59.5 years). Bone (particularly vertebrae), the contralateral lobe, brain, liver and malignant pleural dissemination were the most common sites of metastases. In terms of EGFR mutations, 12 patients $(60 \%)$ harbored the L858R mutation and 8 patients (40\%) harbored the 19 deletion. The average duration of gefitinib therapy at the time of enrollment was 7.4 months (range, 4-38 months). Regarding the optimal treatment response, 1 patient reached complete response, 10 patients reached partial response and 
Table I. Characteristics and clinical data of patients.

\begin{tabular}{|c|c|c|c|c|c|c|c|c|}
\hline No. & $\begin{array}{l}\text { Age/ } \\
\text { sex }\end{array}$ & $\begin{array}{l}\text { Sensitizing } \\
\text { mutation }\end{array}$ & $\begin{array}{l}\text { Primary } \\
\text { foci }\end{array}$ & Metastases & $\begin{array}{l}\text { Optimal } \\
\text { response }\end{array}$ & $\begin{array}{l}\text { Progression } \\
\text { status }\end{array}$ & PFS & $\begin{array}{c}\text { T790M } \\
\text { status }\end{array}$ \\
\hline 1 & $57 / \mathrm{M}$ & 19del & $\begin{array}{l}\text { Superior lobe } \\
\text { of left lung }\end{array}$ & $\begin{array}{l}\text { Liver, brain } \\
\text { and bone }\end{array}$ & PR & Yes & 9 & No \\
\hline 2 & $58 / \mathrm{F}$ & L858R & $\begin{array}{l}\text { Superior lobe } \\
\text { of left lung }\end{array}$ & Bones & PR & No & 8 & No \\
\hline 3 & $43 / \mathrm{F}$ & 19del & Right Lung & Left lung & $\mathrm{CR}$ & No & 16 & Yes \\
\hline 4 & $60 / \mathrm{F}$ & 19del & $\begin{array}{l}\text { Superior lobe } \\
\text { of left lung }\end{array}$ & Right lung & SD & Yes & 13 & Yes \\
\hline 5 & $57 / \mathrm{F}$ & L858R & $\begin{array}{l}\text { Inferior lobe } \\
\text { of right lung }\end{array}$ & Vertebrae & PR & Yes & 13 & No \\
\hline 6 & $70 / \mathrm{F}$ & L858R & $\begin{array}{l}\text { Middle lobe } \\
\text { of right lung }\end{array}$ & Brain & SD & Yes & 29 & Yes \\
\hline 7 & $70 / F$ & L858R & $\begin{array}{l}\text { Inferior lobe } \\
\text { of right lung }\end{array}$ & Left lung & PR & Yes & 12 & No \\
\hline 8 & $62 / F$ & L858R & $\begin{array}{l}\text { Superior lobe } \\
\text { of right lung }\end{array}$ & Left lung & PR & Yes & 15 & No \\
\hline 9 & $74 / \mathrm{F}$ & 19del & Left lung & Bilateral MPE & PR & Yes & 47 & Yes \\
\hline 10 & $64 / \mathrm{F}$ & 19del & Left lung & Right lung & SD & Yes & 11 & No \\
\hline 11 & $72 / \mathrm{F}$ & L858R & $\begin{array}{l}\text { Superior lobe } \\
\text { of left lung }\end{array}$ & Vertebrae & SD & Yes & 24 & Yes \\
\hline 12 & $50 / \mathrm{F}$ & 19del & $\begin{array}{l}\text { Inferior lobe } \\
\text { of left lung }\end{array}$ & $\begin{array}{l}\text { Right lung, } \\
\text { bones }\end{array}$ & PR & Yes & 12 & Yes \\
\hline 13 & $37 / F$ & L858R & $\begin{array}{l}\text { Inferior lobe } \\
\text { of left lung }\end{array}$ & $\begin{array}{l}\text { Bilateral MPE, } \\
\text { bones }\end{array}$ & SD & Yes & 15 & Yes \\
\hline 14 & $53 / \mathrm{F}$ & L858R & $\begin{array}{l}\text { Superior lobe } \\
\text { of left lung }\end{array}$ & Vertebrae & PR & Yes & 10 & Yes \\
\hline 15 & $53 / \mathrm{F}$ & L858R & $\begin{array}{l}\text { Superior lobe } \\
\text { of left lung }\end{array}$ & Vertebrae & SD & Yes & 11 & Yes \\
\hline 16 & $63 / F$ & 19del & Both lungs & Cervical LN & PR & Yes & 6 & No \\
\hline 17 & $67 / \mathrm{F}$ & L858R & Left lung & Cervical LN & PR & Yes & 30 & Yes \\
\hline 18 & $56 / \mathrm{M}$ & L858R & $\begin{array}{l}\text { Inferior lobe } \\
\text { of right lung }\end{array}$ & Bones & SD & Yes & 8 & Yes \\
\hline 19 & 44/M & 19del & $\begin{array}{l}\text { Inferior lobe } \\
\text { of right lung }\end{array}$ & Bones & SD & Yes & 10 & No \\
\hline 20 & $68 / \mathrm{M}$ & L858R & $\begin{array}{l}\text { Inferior lobe } \\
\text { of left lung }\end{array}$ & Bones & SD & Yes & 12 & No \\
\hline
\end{tabular}

No., patient number; 19del, exon 19 E746-A750 deletion; L858R, L858R mutation; PR, partial response; CR, complete response; SD, stable disease; MPE, malignant pleural effusion; LN, lymph nodes.

the remaining 9 patients had stable disease. By the end of the study, 18 patients had disease progression (7 19del patients and 11 L858R patients), with a median PFS of 12.0 months $(95 \%$ CI, 10.3-13.7 months).

Validation of ddPCR assays. To test the analytic sensitivity and specificity of the three ddPCR assays, mutant DNA was serially diluted from NCI-H1650 cells (harboring 19 deletion) and NCIH1975 cells (harboring L858R and T790M mutations) in human reference genomic DNA (catalog no., G1471; Promega Corporation, Madison, WI, USA) in decreasing ratios (1:10 to $1: 10,000)$. The assays were able to detect mutation abundance as low as $0.05 \%$, with a relative error of $<20 \%$. Similarly, by serially decreasing the amount of mutant DNA added, a detection limit of 20 copies/ml of mutant DNA was demonstrated (Fig. 1). With a mutation abundance of $>0.05 \%$ and a mutation concentration of $>20$ copies $/ \mathrm{ml}$, the assays demonstrated linear quantification across a dynamic range spanning 4 orders of magnitude (data not shown).

To determine the reference range of the assays, EGFR mutations were examined in the samples from the 5 healthy donors. Low levels of EGFR mutations were detected, with a peak value of 7 copies $/ \mathrm{ml}, 13$ copies $/ \mathrm{ml}$ and 5 copies $/ \mathrm{ml}$ for the L858R mutation, 19del and T790M mutation, respectively. As 
A

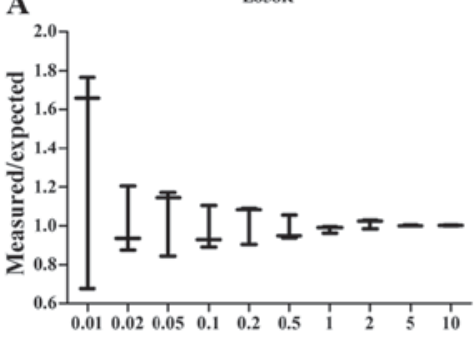

Expected abundance (\%)

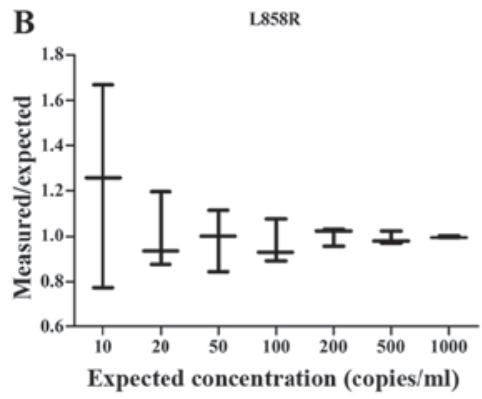

19 del

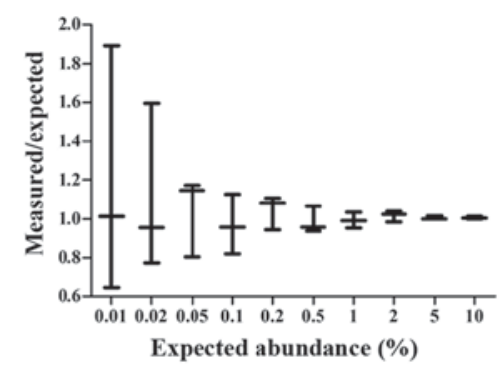

$19 \mathrm{del}$

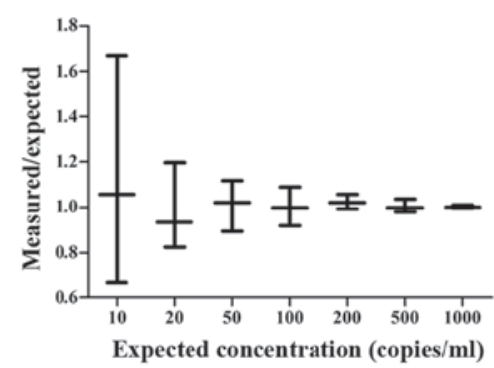

$1790 \mathrm{M}$

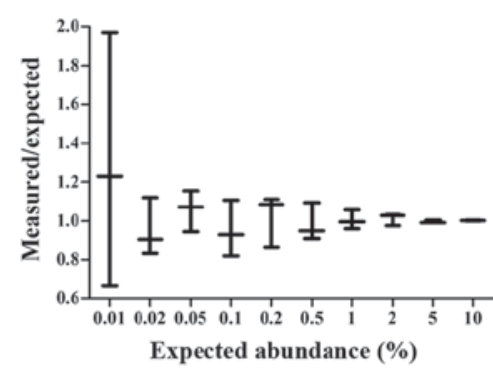

1790M

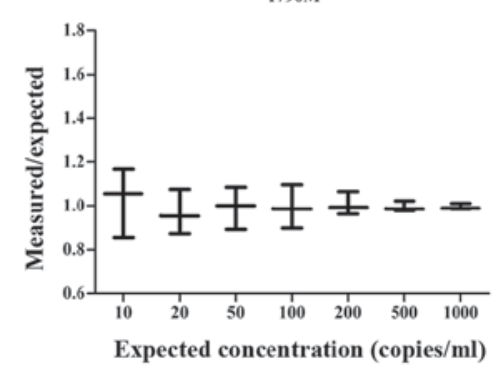

Figure 1. Sensitivity evaluation of epidermal growth factor receptor ddPCR assays. (A) A dilution series of mutant DNA in wild-type DNA in decreasing ratios (1:10 to 1:10,000) was examined in triplicate with the corresponding ddPCR assays (L858R, 19del and T790 M). (B) Serial dilutions of mutant DNA with concentrations ranging from 10-10,0000 copies/ml were examined in triplicate with the corresponding ddPCR assays (L858R, 19del and T790M). The numbers shown in the $\mathrm{x}$-axis represent the expected mutation concentration, while the ratio of measured mutation concentration to expected mutation concentration is displayed in the y-axis. ddPCR, droplet digital polymerase chain reaction.

these detection limits were $<20$ copies/ml, 20 copies/ml was selected as the threshold for a positive result.

EGFR mutations in cfDNA may be quantified for the majority of patients. Each patient enrolled in the present study was followed up monthly for a duration of 4-12 months. Together with the 20 baseline samples, 106 serial plasma samples were collected from 12 L858R patients, and 62 plasma samples were collected from 8 19del patients.

In the baseline samples, corresponding sensitizing mutations were successfully detected in 19 patients (with the exception of patient 7) with an average concentration of 614.8 copies/ml (range, 457.1-1272.7 copies/ml) for L858R patients and 668.9 copies $/ \mathrm{ml}$ (range, $543.7-874.2$ copies $/ \mathrm{ml}$ ) for 19 del patients. Notably, T790M mutations were also detected in 3 patients (patient 15, 18 and 19), with a concentration of 70.21 copies/ml, 98.10 copies $/ \mathrm{ml}$ and 54.37 copies $/ \mathrm{ml}$, respectively.

At the time of disease progression, corresponding sensitizing mutations were detected in all patients, with an average concentration of 572.9 copies $/ \mathrm{ml}$ (range, 192.1-1057.9 copies $/ \mathrm{ml}$ ) for L858R patients and 629.8 copies $/ \mathrm{ml}$ (range, 213.4-965.3copies $/ \mathrm{ml}$ ) for 19del patients. T790M mutations were detected in 10 patients (7 L858R patients and 3 19del patients) with an average concentration of 473.69 copies/ml (range, 106.4-793.8 copies/ml). In addition, EGFR sensitizing mutations and T790M mutations were also detected in patient 2 and patient 3 , whose disease did not reach progression by the end of the present study.

Dynamics of EGFR mutations are associated with treatment response. The serial changes of the EGFR mutation concentrations (L858R or 19del and/or T790M) were generally associated with treatment response observed in images or reflected from clinical manifestations, during or beyond first-line gefitinib therapy (Fig. 2).

Notably, compared with tumor burden (as measured by the sum of diameters of target tumor lesions), the dynamics of EGFR mutations were found to be more helpful in disease monitoring. First, for patients with immeasurable disease (patient 3, 4, 5, 9 and 17), follow-up parameters were otherwise limited. Second, this may also be the case for patients with measurable disease, but presenting exceptional phenomena. The present cohort included patients who had shrinking primary tumors and immeasurable metastatic lesions, including patients with progressive bone metastases confirmed by bone scintigraphy (patient 12; Fig. 1), or accumulating malignant pleural effusion. There were also patients with stable primary disease but sudden metastases, including an emergent brain metastasis that was absent 2 months earlier at the last follow-up, but presented as a considerable tumor mass at the most recent cranial magnetic resonance imaging (patient 14; Fig. 1). For all these patients, the dynamics of EGFR mutations were associated with disease evolution.

Molecular progressive disease may predict drug resistance. Generally, the concentration of sensitizing EGFR mutation dropped upon initiation of gefitinib treatment. In certain patients, the value dropped to zero. Later, this concentration gradually increased during continuous gefitinib treatment. With the exception of the 3 patients with pretreatment T790M mutation, the occurrence of T790M mutation usually accompanied an increase of sensitizing mutation, and the concentration of T790M mutation also continued increasing 
A

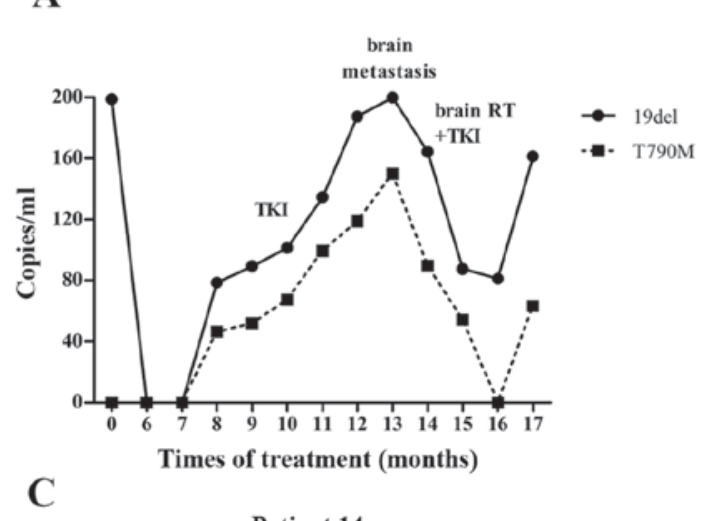

C

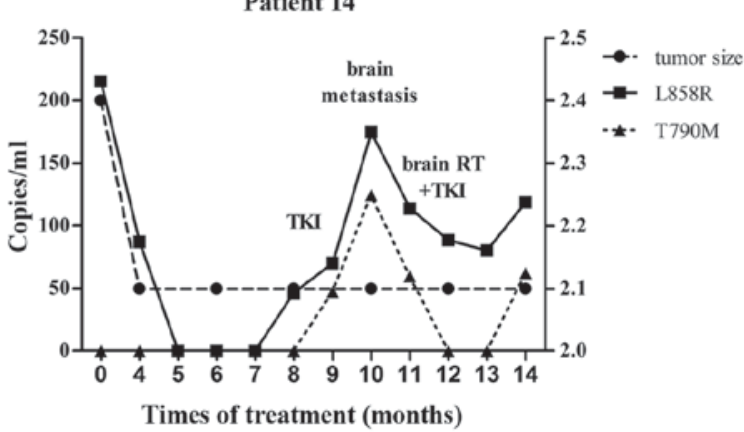

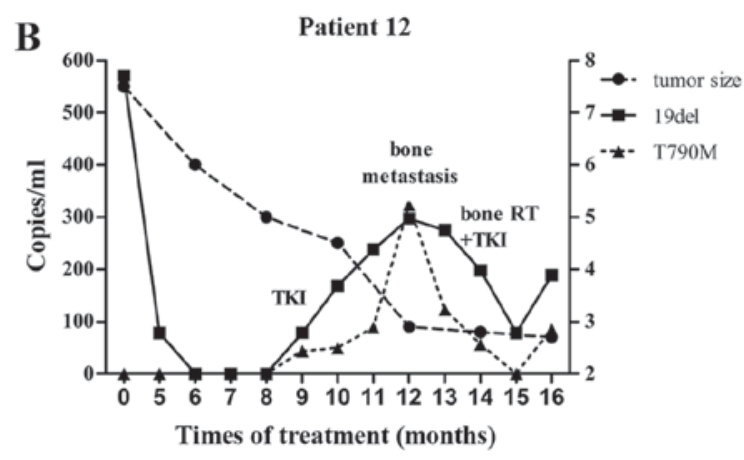

D

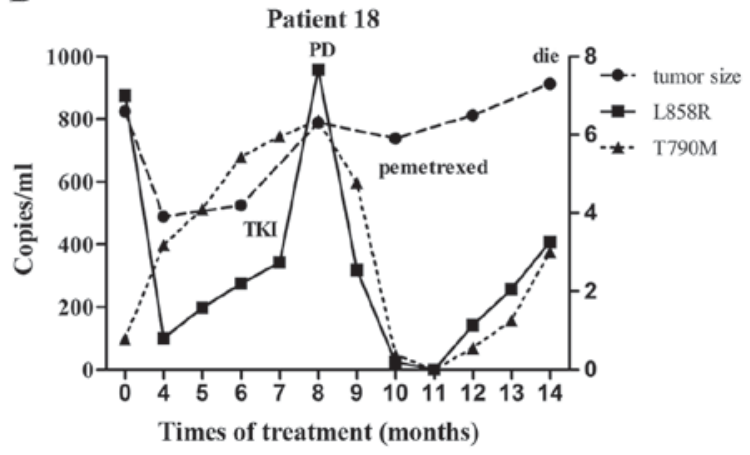

Figure 2. Dynamics of EGFR mutations and disease evolution. (A) Patient 4 was diagnosed with multiple malignant nodules $<1$ cm in the lungs and thus had unmeasurable disease. At 13 months following the initiation of gefitinib therapy, the patient developed a solitary brain metastasis and local radiation was applied. (B) Patient 12 was diagnosed with a large tumor mass in the right lung and multiple bone metastases. The primary tumor mass gradually shrunk during gefitinib therapy, but a new bone metastasis was detected 12 months subsequent to treatment initiation. Local radiation therapy was introduced without discontinuation of gefitinib therapy. (C) Patient 14 was diagnosed with a large malignant nodule in the left lung and multiple bone metastases. The primary tumor lesion was stable during gefitinib therapy, however a solitary brain metastasis emerged 10 months later. Gefitinib therapy was continued and local radiation therapy was added. (D) Patient 18 was diagnosed with multiple tumor masses in the lungs. The tumor masses shrunk in the first several months on gefitinib therapy but continued to grow from the fourth month following treatment initiation. The patient's disease reached radiographic progression after 8 months, and pemetrexed was administered to replace gefitinib therapy. The patient deceased in the 14th month following disease diagnosis. TKI, tyrosine kinase inhibitor; $\mathrm{RT}$, radiation therapy; PD, progression disease.

(Fig. 3). Consequently, molecular progressive disease may be tracked at a median time interval of 4 months (range, 0-8 months) prior to objective progression.

In addition, the dynamics of EGFR mutation may also perform an important role in predicting disease progression under second-line therapy. Specifically, in 5 T790M-positive patients, sensitizing EGFR mutation and T790 M mutation concentration dropped markedly upon the initiation of second-line treatment (local radiation + gefitinib, gefitinib + chemotherapy or changing to chemotherapy), but elevated or reoccurred prior to the second objective progression (Fig. 2).

Prognostic significance of EGFR mutations in cfDNA. In survival analysis, patient PFS was stratified according to age, sex, activating EGFR mutation type (19del vs. L858R), best treatment response to gefitinib (complete response, partial response vs. stable disease), pretreatment tumor size, pretreatment T790M mutation status (pre-T790M+ vs. pre-T790M-), T790M mutation status at the time of disease progression $\left(\mathrm{T} 790 \mathrm{M}^{+}\right.$vs. $\mathrm{T}^{2} 90 \mathrm{M}^{-}$), growth rate of tumor burden and growth rate of EGFR mutation concentrations. Results showed that sex $(\mathrm{P}=0.005)$, pretreatment $\mathrm{T} 790 \mathrm{M}$ mutation status $(\mathrm{P}=0.006), \mathrm{T} 790 \mathrm{M}$ mutation status at the time of disease progression $(\mathrm{P}=0.043)$ and growth rate of EGFR mutation concentration $(\mathrm{P}=0.023)$, were significantly associated with patient survival (Fig. 4).

When examining the prognostic significance of T790M mutation, the results were conflicting. Presence of pretreatment T790M mutation negatively affected patient median PFS $[10.0$ vs. 13.0 months; hazard ration $(\mathrm{HR})=29.38$; $95 \%$ confidence interval (CI), 2.627-328.7], while patients with negative T790M mutation at the time of disease progression had an inferior median PFS (12 months vs. 15 months; HR=3.785; 95\% CI, 1.134-12.62).

In addition, growth rate of EGFR mutation, but not growth rate of tumor burden, was associated with patient survival. First, growth rates of tumor burden were calculated in 15 patients with measurable disease and the median growth rate was $11 \%$ (range, 0-26\%). No significant difference of median PFS stratified by the growth rate of tumor burden was found, no matter which cut-off was selected, including $11 \%$ (the median growth in the present study) or $20 \%$ (proposed in a previous study) (18). The growth rates of EGFR mutation were calculated in all 20 patients and the median growth rate was $50 \%$ (range, $0-101 \%$ ). Using $50 \%$ as a cut-off value, patients with a growth rate $<50 \%$ had a superior median PFS (15.0 months vs. 12.0 months; $\mathrm{HR}=0.251 ; 95 \% \mathrm{CI}, 0.075-0.833$ ) compared with those with a growth rate $>50 \%$. 
A

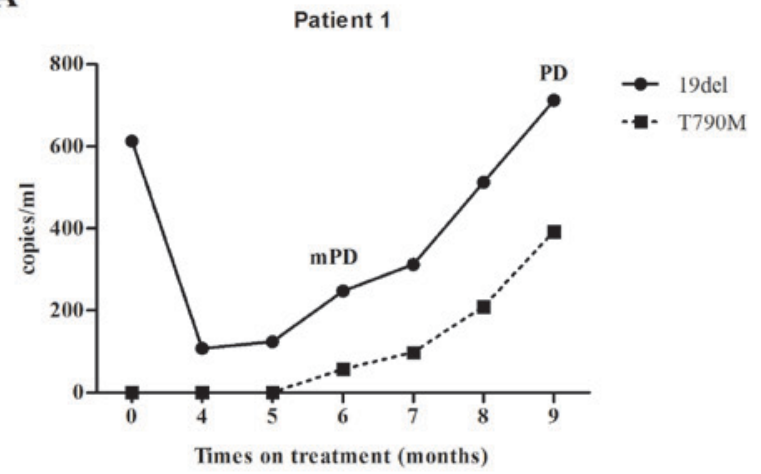

C

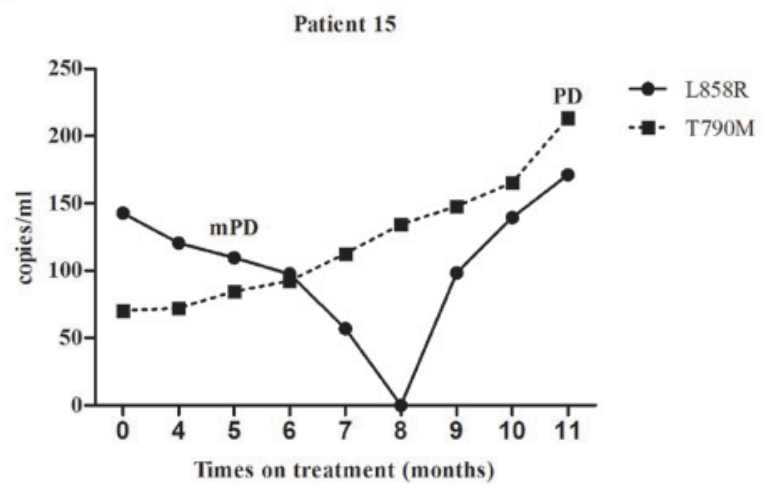

B

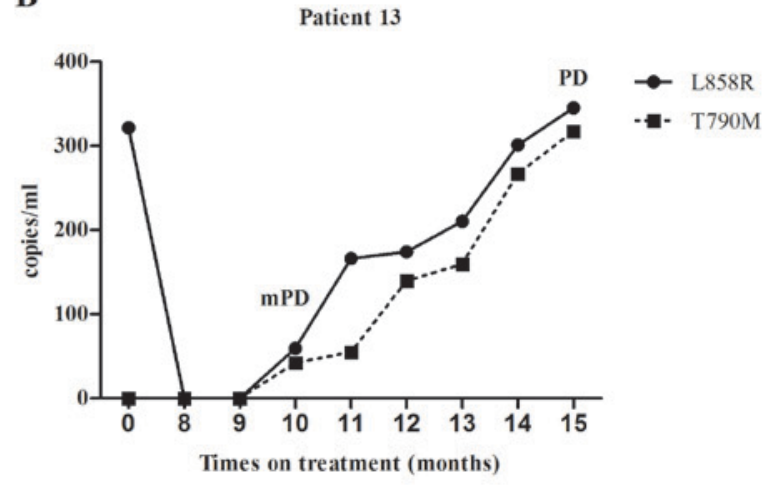

D

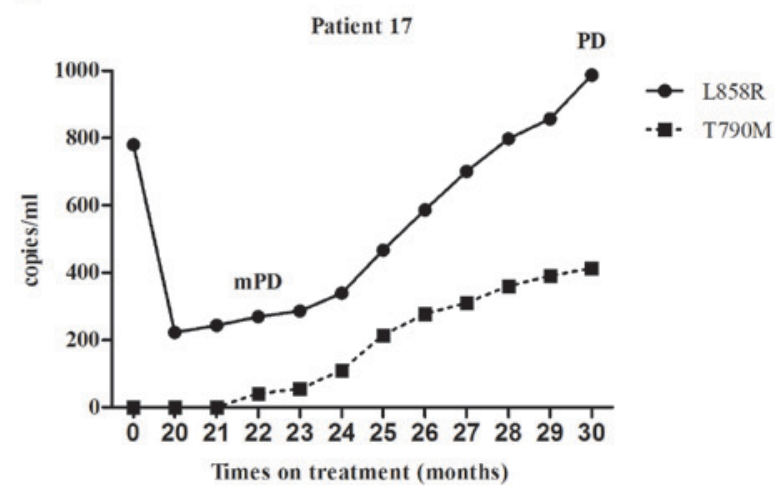

Figure 3. Molecular progression disease, defined as a $\geq 20 \%$ increase of epidermal growth factor receptor mutation concentration compared with the lowest concentration recorded during treatment, usually emerged several months earlier than the documentation of objective progression disease defined by Response Evaluation Criteria in Solid Tumors 1.1. (A) Patient 1 was diagnosed with plasma-positive EGFR 19del mutation and plasma-negative EGFR T790M mutation. On the 6th month of gefitinib treatment, EGFR T790M mutation was detected (demonstrating mPD), accompanied by the elevation of EGFR 19del mutation concentration reaching mPD (the lowest concentration of EGFR 19del mutation was recorded on the 4th month). Development of a new bone metastasis on the 9th month, marked PD. (B) For patient 13, the concentration of plasma EGFR L858R mutation reduced upon the initiation of gefitinib treatment and it was undetectable on the 8th month. However, 2 months later, the activating EGFR mutation (L858R) was re-detected, along with the emergence of EGFR T790M mutation, marking the development of mPD. On the 15th month, a significant re-accumulation of malignant pleural effusion demonstrated PD. (C) Patient 15 was diagnosed with plasma-positive pretreatment EGFR T790M mutation (pretreatment EGFR T790M mutation was undetectable in tumor tissues, using Amplification Refractory Mutation System; and there was inadequate tissue sample for further analysis using droplet digital polymerase chain reaction). During continuous gefitinib treatment, the concentration of EGFR T790M mutation increased, reaching mPD on the 5th month. During the same time, the concentration of the sensitizing EGFR mutation (L858R) was still decreasing. On the 11th month, PD was documented when multiple lesions in the left lung progressed. (D) Elevation of the activating EGFR mutation (L858R) occurred prior to the emergence of the resistant EGFR mutation (T790M) in patient 17, and $\mathrm{mPD}$ was demonstrated on the 22th month of gefitinib treatment. A new lymph node metastasis was noted on the 30 th month, marking PD. mPD, molecular progression disease; PD, progression disease.

\section{Discussion}

Drug resistance to first- and second-generation EGFR TKIs is a major concern for patients with lung cancer harboring activated EGFR mutations. With the emergence of third-generation EGFR TKIs specifically targeting T790M mutation, the development of noninvasive tools for cancer genotyping and disease monitoring is required (7-8). In the present study, EGFR mutations were successfully quantified in cfDNA from 168 monthly collected samples, and their effectiveness in disease monitoring and prognosis characterization was demonstrated. To the best of our knowledge, this is the first prospective cohort that consecutively quantified EGFR mutations in cfDNA for patients with metastatic EGFR-mutant lung adenocarcinoma receiving gefitinib treatment in China.

In line with previous studies $(14-17,19,20)$, the present study confirmed the feasibility and accuracy of quantifying EGFR mutations in cfDNA and found it to be a promising approach for early prediction of drug resistance. Corresponding sensitizing EGFR mutations were correctly detected in 19 (95.0\%) of the 20 patients in the baseline samples, highlighting the ultra-sensitivity of ddPCR $(13,15,17,19-21)$. At the time of disease progression, T790M mutations were detected in 10 out of $18(55.6 \%)$ patients, the frequency of which was comparable to former studies using re-biopsy tissue samples $(6,22,23)$. In addition, molecular progressive disease occurred up to 8 months prior to objective progression, indicating the approach of drug resistance. With the emergence of AZD9291 and CO1686, future clinical trials are warranted to explore the best timing and schedule of these novel EGFR-TKIs in cfDNA-based T790M-positive patients.

Pretreatment T790M mutation or de novo T790M mutation has been observed in tumor tissues using ultra-sensitive detection platforms, with an incidence up to $79 \%$ (24-26). In 
A

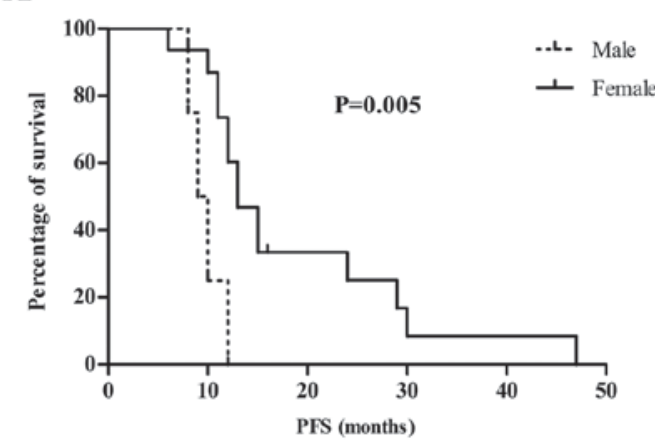

C

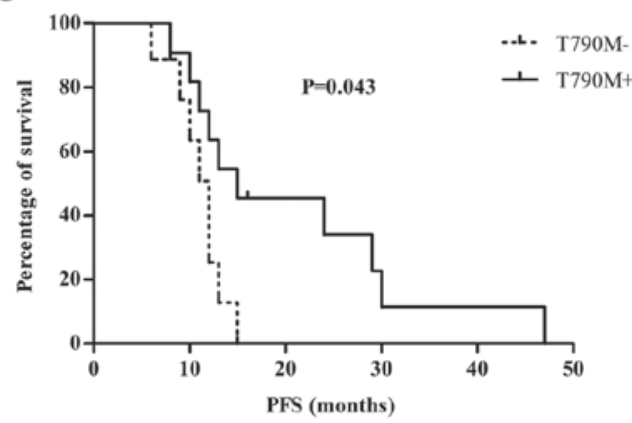

B

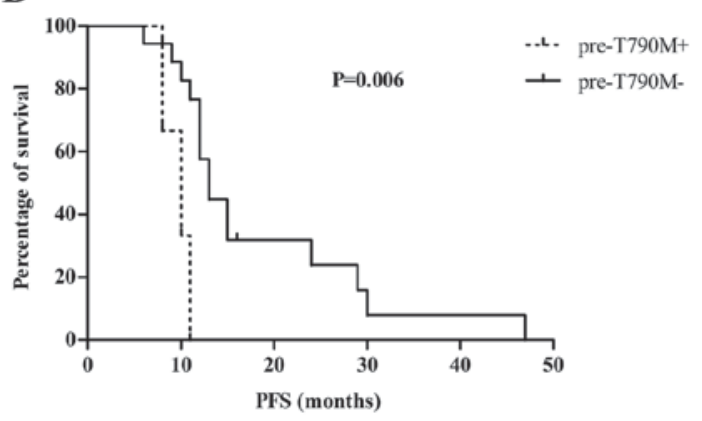

D

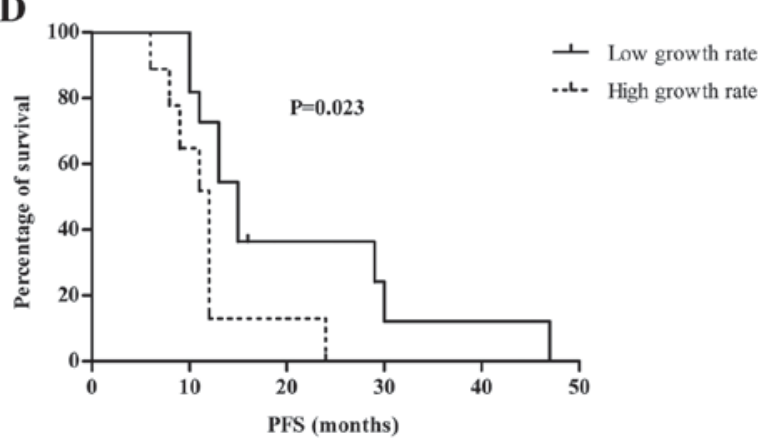

Figure 4. Sex, T790M mutation status, growth rate of EGFR mutations and survival. (A) Male patients had a significantly shorter PFS compared with female counterparts (9.5 vs. 13.0 months, $\mathrm{P}=0.005 ; \mathrm{HR}, 16.62 ; 95 \% \mathrm{CI}, 2.308-119.7)$. (B) Presence of pretreatment T790M mutation negatively affected patient median PFS (10.0 vs. 13.0 months, $\mathrm{P}=0.006$; HR, 29.38; 95\% CI, 2.627-328.7). (C) Absence of T790M mutation at the time of disease progression negatively affected patient median PFS (12 vs. 15 months, $\mathrm{P}=0.043$; HR, 3.785; 95\% CI, 1.134-12.62). (D) Patients with a low growth rate (monthly growth rate <50\%) of EGFR mutation concentrations in cell-free DNA had a superior median PFS (15.0 vs. 12.0 months, P=0.023; HR, 0.251; 95\% CI, 0.075-0.833) compared with those having a high growth rate (monthly growth rate $>50 \%$ ). PFS, progression-free survival; HR, hazard ratio; CI, confidence interval; EGFR, epidermal growth factor receptor.

the present study, patients with negative T790M mutations in their pretreatment tissues, confirmed by ARMS, were enrolled. However, pretreatment T790M mutation in cfDNA was detected in $3(15 \%)$ cases. The different sensitivity between ARMS and ddPCR may explain this disparity. The sensitivity of detecting EGFR mutations using ARMS is $1 \%$ (27), and thus, mutations with frequencies $<1 \%$ may end up with false-negative results. For surgically resected tumor samples, pretreatment T790M mutation commonly presented at a low ratio $(<0.1 \%)(26)$. To determine the incidence and abundance of de novo T790M in advanced patients with lung cancer, quantifying pretreatment T790M mutation in tissue samples and cfDNA using ultra-sensitive detection methods, including ddPCR, is recommended.

In addition, the presence of T790M mutation in cfDNA prior to the initiation of EGFR TKIs as a second-line therapy has been significantly associated with a shorter PFS (28). Similarly, positive pretreatment T790M mutation, in the present study, was found to negatively affect patient PFS under first-line gefitinib therapy. These observations raise questions regarding the best regimen for this subgroup of patients. Whether third-generation EGFR TKIs, including AZD9291, should be administered in the first-line or used until the abundance of T790M mutation reaches a certain threshold, is now an open question. Additional well-designed randomized controlled trials are required to answer these questions.
In the current study, although the presence of pretreatment T790M mutation negatively affected patient PFS, presence of T790M mutation at the time of disease progression had the opposite effect. There are multiple mechanisms underlying drug resistance to EGFR TKIs, and numerous studies have identified that presence of T790M mutation at time of disease progression positively affects patient survival, regardless of whether T790M mutation is detected in re-biopsy tissues or in cfDNA, or whether the prognosis is presented as median PFS, median overall survival or 5-year survival rate (21-24). One of the explanations may come from the in vitro study that demonstrated EGFR-mutated T790M-positive cells have a slower proliferation rate compared with T790M-negative cells (29).

Circulating tumor DNA in the plasma may be passively accumulated or actively released and may reflect the systemic tumor burden and the overall tumor activity $(30,31)$. The dynamics of EGFR mutation concentrations were associated with treatment response in almost every patient in the present study, highlighting the potential role of cfDNA-based parameters in disease monitoring. Notably, the dynamics of EGFR mutations were consistent with disease evolution, even in patients with immeasurable disease and patients with exceptional phenomena. In these patients, estimating tumor burden using bimonthly radiographic examinations may be misleading, and thus quantifying EGFR mutations in cfDNA may be a valuable alternative or at least adjuvant. 
In addition to disease monitoring, parameters derived from cfDNA-based mutation quantification may also have prognostic significance. It has been well recognized that tumor burden expressed as the number of metastatic sites, uptake of ${ }^{18} \mathrm{~F}$-fluorodeoxyglucose measured by positron emission tomography, or tumor volume analyzed using special software associated well with patient survival (32-35). However, the prognostic significance of genetic mutation concentrations in cfDNA is largely unknown $(21,34)$. In the present study, it was discovered that a growth rate of EGFR mutations $>50 \%$ negatively affected patient median PFS, and this observation requires validation in larger cohorts.

\section{Acknowledgements}

The authors thank Paul Horak from the Johns Hopkins University School of Medicine and Professor Zhang Li from Sun Yat-Sen University Cancer Center for reading the manuscript. The present study was supported by Wu Jieping Medical Foundation (grant no. 320.6750 .14281$)$.

\section{References}

1. Mok TS, Wu YL, Thongprasert S, Yang CH, Chu DT, Saijo N, Sunpaweravong P, Han B, Margono B, Ichinose Y, et al: Gefitinib or carboplatin-paclitaxel in pulmonary adenocarcinoma. N Engl J Med 361: 947-957, 2009.

2. Maemondo M, Inoue A, Kobayashi K, Sugawara S, Oizumi S, Isobe H, Gemma A, Harada M, Yoshizawa H, Kinoshita I, et al: Gefitinib or chemotherapy for non-small-cell lung cancer with mutated EGFR. N Engl J Med. 362: 2380-2388, 2010.

3. Mitsudomi T, Morita S, Yatabe Y, Negoro S, Okamoto I, Tsurutani J, Seto T, Satouchi M, Tada H, Hirashima T, et al: Gefitinib versus cisplatin plus docetaxel in patients with non-small-cell lung cancer harbouring mutations of the epidermal growth factor receptor (WJTOG3405): An open label, randomised phase 3 trial. Lancet Oncol 11: 121-128, 2010.

4. Kobayashi S, Boggon TJ, Dayaram T, Jänne PA, Kocher O, Meyerson M, Johnson BE, Eck MJ, Tenen DG and Halmos B: EGFR mutation and resistance of non-small-cell lung cancer to gefitinib. N Engl J Med 352: 786-792, 2005.

5. Maheswaran S, Sequist LV, Nagrath S, Ulkus L, Brannigan B, Collura CV, Inserra E, Diederichs S, Iafrate AJ, Bell DW, et al: Detection of mutations in EGFR in circulating lung-cancer cells. N Engl J Med 359: 366-377, 2008.

6. Yu HA, Arcila ME, Rekhtman N, Sima CS, Zakowski MF, Pao W, Kris MG, Miller VA, Ladanyi M and Riely GJ: Analysis of tumor specimens at the time of acquired resistance to EGFR-TKI therapy in 155 patients with EGFR-mutant lung cancers. Clin Cancer Res 19: 2240-2247, 2013.

7. Jänne PA, Yang JC, Kim DW, Planchard D, Ohe Y, Ramalingam SS, Ahn MJ, Kim SW, Su WC, Horn L, et al: AZD9291 in EGFR inhibitor-resistant non-small-cell lung cancer. N Engl J Med 372: 1689-1699, 2015.

8. Sequist LV, Soria JC, Goldman JW, Wakelee HA, Gadgeel SM, Varga A, Papadimitrakopoulou V, Solomon BJ, Oxnard GR, Dziadziuszko R, et al: Rociletinib in EGFR-mutated non-small-cell lung cancer. N Engl J Med 372: 1700-1709, 2015.

9. Murtaza M, Dawson SJ, Tsui DW, Gale D, Forshew T, Piskorz AM, Parkinson C, Chin SF, Kingsbury Z, Wong AS, et al: Non-invasive analysis of acquired resistance to cancer therapy by sequencing of plasma DNA. Nature 497: 108-112, 2013.

10. Del Re M, Vasile E, Falcone A, Danesi R and Petrini I: Molecular analysis of cell-free circulating DNA for the diagnosis of somatic mutations associated with resistance to tyrosine kinase inhibitors in non-small-cell lung cancer. Expert Rev Mol Diagn 14: 453-468, 2014.

11. Tseng JS, Yang TY, Tsai CR, Chen KC, Hsu KH, Tsai MH, Yu SL, Su KY, Chen JJ and Chang GC: Dynamic plasma EGFR mutation status as a predictor of EGFR-TKI efficacy in patients with EGFR-mutant lung adenocarcinoma. J Thorac Oncol 10: 603-610, 2015
12. Ottesen EA, Hong JW, Quake SR and Leadbetter JR: Microfluidic digital PCR enables multigene analysis of individual environmental bacteria. Science 314: 1464-1467, 2006.

13. Hindson CM, Chevillet JR, Briggs HA, Gallichotte EN, Ruf IK, Hindson BJ, Vessella RL and Tewari M: Absolute quantification by droplet digital PCR versus analog real-time PCR. Nat Methods 10: 1003-1005, 2013.

14. Yung TK, Chan KC, Mok TS, Tong J, To KF and Lo YM: Single-molecule detection of epidermal growth factor receptor mutations in plasma by microfluidics digital PCR in non-small cell lung cancer patients. Clin Cancer Res 15: 2076-2084, 2009.

15. Oxnard GR, Paweletz CP, Kuang Y, Mach SL, O'Connell A, Messineo MM, Luke JJ, Butaney M, Kirschmeier P, Jackman DM and Jänne PA: Noninvasive detection of response and resistance in EGFR-mutant lung cancer using quantitative next-generation genotyping of cell-free plasma DNA. Clin Cancer Res 20: 1698-1705, 2014.

16. Watanabe M, Kawaguchi T, Isa S, Ando M, Tamiya A, Kubo A, Saka H, Takeo S, Adachi H, Tagawa T, et al: Ultra-sensitive detection of the pretreatment EGFR T790M mutation in non-small cell lung cancer patients with an EGFR-activating mutation using droplet digital PCR. Clin Cancer Res 21: 3552-3560, 2015.

17. Zhu G, Ye X, Dong Z, Lu YC, Sun Y, Liu Y, McCormack R, Gu Y and Liu X: Highly sensitive droplet digital PCR method for detection of EGFR activating mutations in plasma cell-free DNA from patients with advanced non-small cell lung cancer. J Mol Diagn 17: 265-272, 2015.

18. Cha YK, Lee HY, Ahn MJ, Choi YL, Lee JH, Park K and Lee KS: Survival outcome assessed according to tumor burden and progression patterns in patients with epidermal growth factor receptor mutant lung adenocarcinoma undergoing epidermal growth factor receptor tyrosine kinase inhibitor therapy. Clin Lung Cancer 16: 228-236, 2015.

19. Sorensen BS, Wu L, Wei W, Tsai J, Weber B, Nexo E and Meldgaard P: Monitoring of epidermal growth factor receptor tyrosine kinase inhibitor-sensitizing and resistance mutations in the plasma DNA of patients with advanced non-small cell lung cancer during treatment with erlotinib. Cancer 120: 3896-3901, 2014.

20. Ishii H, Azuma K, Sakai K, Kawahara A, Yamada K, Tokito T, Okamoto I, Nishio K and Hoshino T: Digital PCR analysis of plasma cell-free DNA for non-invasive detection of drug resistance mechanisms in EGFR mutant NSCLC: Correlation with paired tumor samples. Oncotarget 6: 30850-30858, 2015.

21. Wang Z, Chen R, Wang S, Zhong J, Wu M, Zhao J, Duan J, Zhuo M, An T, Wang Y, et al: Quantification and dynamic monitoring of EGFR T790M in plasma cell-free DNA by digital PCR for prognosis of EGFR-TKI treatment in advanced NSCLC. PLoS One 9: e110780, 2014.

22. Kuiper JL, Heideman DA, Thunnissen E, Paul MA, van Wijk AW, Postmus PE and Smit EF: Incidence of T790M mutation in (sequential) rebiopsies in EGFR-mutated NSCLC-patients. Lung Cancer 85: 19-24, 2014.

23. Hata A, Katakami N, Yoshioka H, Takeshita J, Tanaka K, Nanjo S, Fujita S, Kaji R, Imai Y, Monden K, et al: Rebiopsy of non-small cell lung cancer patients with acquired resistance to epidermal growth factor receptor-tyrosine kinase inhibitor: Comparison between T790M mutation-positive and mutation-negative populations. Cancer 119: 4325-4332, 2013.

24. Su KY, Chen HY, Li KC, Kuo ML, Yang JC, Chan WK, Ho BC, Chang GC, Shih JY, Yu SL and Yang PC: Pretreatment epidermal growth factor receptor (EGFR) T790M mutation predicts shorter EGFR tyrosine kinase inhibitor response duration in patients with non-small-cell lung cancer. J Clin Oncol 30: 433-440, 2012 .

25. Fujita Y, Suda K, Kimura H, Matsumoto K, Arao T, Nagai T, Saijo N, Yatabe Y, Mitsudomi T and Nishio K: Highly sensitive detection of EGFR T790M mutation using colony hybridization predicts favorable prognosis of patients with lung cancer harboring activating EGFR mutation. J Thorac Oncol 7: 1640-1644, 2012.

26. Watanabe M, Kawaguchi T, Isa SI, Ando M, Tamiya A, Kubo A, Saka H, Takeo S, Adachi H, Tagawa T, et al: Ultra-sensitive detection of the pretreatment EGFR T790M mutation in non-small-cell lung cancer patients with an EGFR-activating mutation using droplet digital PCR. Clin Cancer Res 21: 3552-3560, 2015.

27. Zhou Q, Zhang XC, Chen ZH, Yin XL, Yang JJ, Xu CR, Yan HH, Chen HJ, Su J, Zhong WZ, et al: Relative abundance of EGFR mutations predicts benefit from gefitinib treatment for advanced non-small-cell lung cancer. J Clin Oncol 29: 3316-3321, 2011. 
28. Zheng D, Ye X, Zhang MZ, Sun Y, Wang JY, Ni J, Zhang HP, Zhang L, Luo J, Zhang J, et al: Plasma EGFR T790M ctDNA status is associated with clinical outcome in advanced NSCLC patients with acquired EGFR-TKI resistance. Sci Rep 6: 20913, 2016.

29. Chmielecki J, Foo J, Oxnard GR, Hutchinson K, Ohashi K, Somwar R, Wang L, Amato KR, Arcila M, Sos ML, et al: Optimization of dosing for EGFR-mutant non-small cell lung cancer with evolutionary cancer modeling. Sci Transl Med 3: 90ra59, 2011.

30. Crowley E, Di Nicolantonio F, Loupakis F and Bardelli A: Liquid biopsy: Monitoring cancer-genetics in the blood. Nat Rev Clin Oncol 10: 472-484, 2013

31. Haber DA and Velculescu VE: Blood-based analyses of cancer: Circulating tumor cells and circulating tumor DNA. Cancer Discov 4: 650-661, 2014.

32. Park JH, Kim TM, Keam B, Jeon YK, Lee SH, Kim DW, Chung DH, Kim YT, Kim YW and Heo DS: Tumor burden is predictive of survival in patients with non-small-cell lung cancer and with activating epidermal growth factor receptor mutations who receive gefitinib. Clin Lung Cancer 14: 383-389, 2013.
33. Zander T, Scheffler M, Nogova L, Kobe C, Engel-Riedel W, Hellmich M,Papachristou I, Toepelt K, Draube A, Heukamp L, et al: Early prediction of nonprogression in advanced non-small-cell lung cancer treated with erlotinib by using [(18)F]fluorodeoxyglucose and [(18)F]fluorothymidine positron emission tomography. J Clin Oncol 29: 1701-1708, 2011.

34. Mok T, Wu YL, Lee JS, Yu CJ, Sriuranpong V, Sandoval-Tan J, Ladrera G, Thongprasert S, Srimuninnimit V, Liao M, et al: Detection and dynamic changes of EGFR mutations from circulating tumor DNA as a predictor of survival outcomes in NSCLC patients treated with first-line intercalated erlotinib and chemotherapy. Clin Cancer Res 21: 3196-3203, 2015.

35. Nishino M, Dahlberg SE, Cardarella S, Jackman DM, Rabin MS, Ramaiya NH, Hatabu H, Jänne PA and Johnson BE: Volumetric tumor growth in advanced non-small cell lung cancer patients with EGFR mutations during EGFR-tyrosine kinase inhibitor therapy: Developing criteria to continue therapy beyond RECIST progression. Cancer 119: 3761-3768, 2013. 